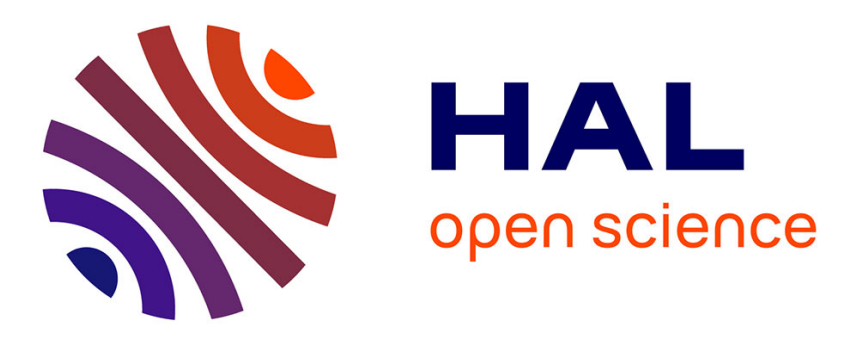

\title{
Fast diagnostic test for familial Mediterranean fever based on a kinase inhibitor
}

Flora Magnotti, Tiphaine Malsot, Sophie Georgin-Lavialle, Fatima Abbas, Amandine Martin, Alexandre Belot, Maxime Fauter, Muriel Rabilloud, Mathieu Gerfaud-Valentin, Pascal Sève, et al.

\section{To cite this version:}

Flora Magnotti, Tiphaine Malsot, Sophie Georgin-Lavialle, Fatima Abbas, Amandine Martin, et al.. Fast diagnostic test for familial Mediterranean fever based on a kinase inhibitor. Annals of the Rheumatic Diseases, 2020, pp.annrheumdis-2020-218366. 10.1136/annrheumdis-2020-218366 . hal02963374

\section{HAL Id: hal-02963374 \\ https://hal.science/hal-02963374}

Submitted on 11 Jun 2021

HAL is a multi-disciplinary open access archive for the deposit and dissemination of scientific research documents, whether they are published or not. The documents may come from teaching and research institutions in France or abroad, or from public or private research centers.
L'archive ouverte pluridisciplinaire HAL, est destinée au dépôt et à la diffusion de documents scientifiques de niveau recherche, publiés ou non, émanant des établissements d'enseignement et de recherche français ou étrangers, des laboratoires publics ou privés. 


\section{${ }^{1} \mathrm{~A}$ fast diagnostic test for familial Mediterranean fever based on a}

\section{kinase inhibitor}

Flora Magnotti ${ }^{1}$, Tiphaine Malsot ${ }^{1}$, Sophie Georgin-Lavialle ${ }^{2}$, Fatima Abbas ${ }^{3}$, Amandine Martin ${ }^{1}$, Alexandre Belot $^{1,4}$, Maxime Fauter ${ }^{1,5}$, Muriel Rabilloud 3 , Mathieu Gerfaud-Valentinn ${ }^{5}$ Pascal Sève ${ }^{5}$, Agnès Duquesne ${ }^{4}$, Arnaud Hot ${ }^{6}$, Stéphane Durupt ${ }^{7}$, Léa Savey², Irina Giurgea ${ }^{8}$, Gilles Grateau ${ }^{2}$, Thomas Henry ${ }^{1, \#, ~ a n d ~ Y v a n ~}$ Jamilloux ${ }^{1,5, \#}$

\footnotetext{
${ }^{1}$ CIRI, Centre International de Recherche en Infectiologie, Inserm U1111 ; CNRS, UMR5308 ; ENS de Lyon, Université Claude Bernard Lyon 1, Lyon, France

${ }^{2}$ Service de Médecine Interne, Hôpital Tenon, APHP ; CEREMAIA (Centre de Référence des Maladies Autoinflammatoires et des Amyloses), Paris, France

${ }^{3}$ Pôle Santé Publique, Service de Biostatistique et Bioinformatique ; CNRS, UMR 5558, Laboratoire de Biométrie et Biologie Évolutive, Équipe Biostatistique-Santé ; Hospices Civils de Lyon ; Université de Lyon, Lyon, France

${ }^{4}$ Service de Rhumato-Néphro-Dermatologie pédiatrique, Hôpital Femme-Mère-Enfants, Hospices Civils de Lyon, Université Claude Bernard Lyon 1 ; CEREMAIA (Centre de Référence des Maladies Autoinflammatoires et des Amyloses), Bron, France

${ }^{5}$ Service de Médecine Interne, Hôpital de la Croix-Rousse, Hospices Civils de Lyon, Université Claude Bernard Lyon 1, Lyon, France

${ }^{6}$ Service de Médecine Interne, Hôpital Edouard Herriot, Hospices Civils de Lyon, Université Claude Bernard Lyon 1 ; CEREMAIA (Centre de Référence des Maladies Autoinflammatoires et des Amyloses), Lyon, France

${ }^{7}$ Service de Médecine Interne, Centre Hospitalier Lyon Sud, Hospices Civils de Lyon, Université Claude Bernard Lyon 1, Lyon, France ${ }^{8}$ Medical Genetics Department, Armand-Trousseau Hospital, APHP, Sorbonne University, Paris, France

\# Co-senior authors
}

Short title: A fast diagnostic test for FMF

Corresponding author: Dr. Yvan Jamilloux, Service de Médecine Interne, Hôpital de la CroixRousse, 103 grande rue de la Croix-Rousse, F-69004, Lyon, France Tel: +33 426732636 - FAX: +33 426732637 - E-mail: yvan.jamilloux@chu-lyon.fr 


\section{ABSTRACT}

Background and Objective Familial Mediterranean fever (FMF) is the most frequent hereditary autoinflammatory disease. Its diagnosis relies on a set of clinical criteria and a genetic confirmation upon identification of bi-allelic pathogenic $M E F V$ variants. $M E F V$ encodes pyrin, an inflammasome sensor. Using a kinase inhibitor, UCN-01, we recently identified that dephosphorylation of FMF-associated pyrin mutants leads to inflammasome activation. The aim of this study was to assess whether quantifying UCN01-mediated inflammasome activation could discriminate FMF patients from healthy donors (HD) and from patients with other inflammatory disorders (OID).

Methods Real time pyroptosis and IL-1 $\beta$ secretion were monitored in response to UCN01 in monocytes from FMF patients $(n=67)$, HD $(n=71)$, and OID patients $(n=40)$. Sensitivity and specificity of the resulting diagnostic tests were determined by ROC curve analyses.

Results Inflammasome monitoring in response to UCN-01 discriminates FMF patients from other individuals. Pyroptosis assessment leads to a fast FMF diagnosis while combining pyroptosis and IL-1 $\beta$ dosage renders UCN-01-based assays highly sensitive and specific. UCN-01-triggered monocytes responses were influenced by MEFV gene dosage and $M E F V$ mutations in a similar way as clinical phenotypes are.

Conclusions UCN-01-based inflammasome assays could be used to rapidly diagnose FMF, with high sensitivity and specificity.

Keywords: Familial Mediterranean fever, pyrin, diagnosis, pyroptosis, interleukin-1 


\section{INTRODUCTION}

Familial Mediterranean fever (FMF) is the most common inherited autoinflammatory syndrome. ${ }^{1}$ FMF is present worldwide as a rare disease. A high prevalence (up to $1 / 500$ ) is observed in the Mediterranean basin. ${ }^{2}$ FMF is typically characterized by recurrent attacks of fever and serositis, associated with systemic inflammation. The major challenge of FMF is to establish a fast and definitive diagnosis, to avoid unnecessary and costly investigations, prolonged diagnostic wandering, or useless life-long treatment. ${ }^{3}$

FMF diagnosis results from a combination of clinical criteria ${ }^{4}$, and is confirmed when biallelic mutations in $M E F V$, the gene encoding pyrin, are observed.5,6 Yet, genetic tests may be inconclusive, about one-third of patients bearing only one mutated $M E F V$ allele. $^{7}$ Genetic analyses can also reveal variants of uncertain significance. ${ }^{8}$ Therefore, it is of great importance to develop a rapid diagnostic test.

Recently, using a kinase inhibitor, UCN-01, we demonstrated that pyrin dephosphorylation triggers full inflammasome activation in FMF patients' monocytes while it does not in healthy donor (HD) monocytes. ${ }^{9}$ We hypothesized that these differential responses could be the basis of a diagnostic test to quickly distinguish FMF patients from HD or patients suffering from other inflammatory disorders (OID).

\section{METHODS}

Methods are detailed in the supplementary material.

The study was approved by the French Comité de Protection des Personnes (\#2018/95). Every participant (supplementary table 1) gave informed consent. Statistical analysis was performed with R software and P-values $<0.05$ were considered significant. 


\section{RESULTS}

\section{Cell death kinetics discriminates FMF patients from HD and patients with OID}

Pyroptosis was monitored in real time in monocytes from FMF patients bearing biallelic p.M694V or p.M694I mutations, HD, and controls with OID. UCN-01 triggered a rapid cell death in FMF patients' monocytes while it was much delayed in monocytes from HD or OID (figure 1A). This difference in cell death kinetics was highly significant as determined both by quantifying the areas under the curve (AUC, $p<0.0001$; figure $1 B$ ) and by comparing the UCN-01-incubation time leading to $20 \%$ of cell death $(\mathrm{p}<0.0001$; figure 1C). ROC curves were generated and regression analysis established that monitoring cell death during 60 minutes of UCN-01 treatment discriminated FMF patients from HD with a sensitivity of $95.7 \%$ and a specificity of $94.7 \%$ (figure 1D).

When applied to a cohort of patients with OID, the same analysis accurately classified 94.9\% of the patients. Thus, monitoring UCN-01-triggered pyroptosis discriminates FMF patients from other patients with a sensitivity of $92.1 \%$ and a specificity of $97.5 \%$ (figure 1E).

Of note, this experiment does not require any LPS priming step, providing results within one hour post-monocyte isolation.

\section{Biparametric analyses increase the sensitivity and specificity of the test}

We then wondered whether a biparametric test, based also on the IL-1 $\beta$ release quantification, could better discriminate the different patient groups.

Following LPS priming and pyrin activation, mean IL-1 $\beta$ levels in monocyte supernatants were 15 -fold higher in homozygous FMF patients $(1521 \pm 1168 \mathrm{pg} / \mathrm{mL})$ than in HD $(92.7 \pm 111 \mathrm{pg} / \mathrm{mL} ; \mathrm{p}<0.0001)$ or patients with OID $(99.16 \pm 108 \mathrm{pg} / \mathrm{mL}$; $\mathrm{p}<0.0001$ ) (figure 2A). By comparing these FMF patients with HD, the discrimination 
threshold was determined at $260.6 \mathrm{pg} / \mathrm{mL}$, giving a sensitivity of $97.1 \%$ and a specificity of $91.2 \%$ (figure $2 \mathrm{~B}$ ).

Similar results were obtained when applying this analysis to a cohort of patients with OID (sensitivity of $100 \%$ and a specificity of $97.3 \%$; figure $2 \mathrm{C}$ ). Thus, IL-1 $\beta$ dosage following UCN-01 treatment discriminates FMF patients from HD and other patients with OID.

Importantly, by combining the two parameters, homozygous FMF patients were fully segregated from HD $(\mathrm{p}<0.0001)$, while only one patient in the OID group was classified as a false positive $(\mathrm{p}<0.0001)$ (figure 2D). Of note, the $M E F V$ genotype of this patient

with Behçet disease is unknown and we cannot exclude the possibility that he is carrying a pathogenic $M E F V$ variant (more prevalent in patients with Behçet disease than in an ethnically-matched population). ${ }^{10,11}$

Hence, monitoring both pyroptosis and IL-1 $\beta$ strengthens the discriminating power of the assay (sensitivity of $100 \%$ and specificity of $99 \%$; figure $2 \mathrm{E}-\mathrm{F}$ ).

\section{Gene dosage determines UCN-01-mediated responses}

$M E F V$-gene dosage plays an important role in the phenotype of FMF.12,13 We therefore wondered whether cellular responses to UCN-01 were influenced by gene dosage in the same way as the clinical phenotype. Patients with mono- $(n=10)$ or bi-allelic $(n=38)$ variants at the p.M694 residue were selected for this analysis. Real-time pyroptosis monitoring identified significant differences between heterozygous and homozygous patients ( $p=0.0015$; figure 3A, B). IL-1 $\beta$ analysis revealed significantly lower cytokine levels in heterozygous patients than in homozygous patients $(p=0.024$; figure $3 C)$. Biparametric analyses confirmed the gene dosage effect by demonstrating a significant 
difference between monocytes from carriers of mono- vs. biallelic $M E F V$ variants $(\mathrm{p}=0.0044 ;$ figure 3D).

Importantly, heterozygous FMF patients were also discriminated from HD using UCN01-based assays ( $\mathrm{p}<0.001$; figure $3 \mathrm{~A}-\mathrm{C})$.

Together, these results demonstrate a gene dosage impact on the UCN-01-triggered responses and show that UCN-01-based functional assays segregate homozygous or heterozygous FMF patients from HD. Interestingly, these results are highly consistent with the impact of the $M E F V$ gene dosage on clinical phenotypes. ${ }^{12}$ Of note, colchicine treatment did not impact the results of the test (supplementary figure 1).

\section{$M E F V$ genotype influences monocyte responses to UCN-01}

$374 M E F V$ variants are listed in the Infevers registry and the pathogenicity of most of them is unclear. ${ }^{8,14}$ We thus investigated the responses of monocytes from FMF patients with homozygous mutations not located at the p.M694 site. Three patients with homozygous mutations (p.M680I, p.V726A, and p.P369S) were analyzed (supplementary figure 2A-D). Although caution must be raised due to the inclusion of a single patient per genotype, a gradient of UCN-01-triggered responses was observed which is highly consistent with the described impact of the corresponding mutations on clinical phenotypes. ${ }^{15-17}$ The impact of genotypes on in vitro phenotypes could be classified as follows p.M694V/I>p.M680I >p.V726A>p.P369S which mirrors the clinical phenotype-genotype studies. ${ }^{15-18}$ The biparametric analysis segregated the homozygous p.M680I and p.V726A patients from the HD, but not the FMF patient harboring the p.P369S/P369S genotype (supplementary figure 2D). Interestingly, p.P369S is a variant of uncertain significance which does not behave like typical FMF-associated MEFV variants, as observed in in vitro experiments on monocyte cell lines ${ }^{11}$, or in a recently 
developed colchicine-resistance assay. ${ }^{19}$ These results suggest that the p.P369S variant is a non-pathogenic variant or that its pathogenicity is associated with another molecular mechanism.

Finally, we analyzed the ability of UCN-01-based tests to discriminate compound heterozygous FMF patients from HD (supplementary figure 2E-H). The in vitro responses of monocytes bearing two different $M E F V$ variants were lower than in vitro responses of p.M694V/I homozygous monocytes. When combined with the clearly pathogenic p.M694 variants, mutations on the second $M E F V$ allele impacted differentially the in vitro responses with a hierarchy (p.M680I, p.R761H > p.V726A, p.E148Q) largely mirroring the gradient of clinical phenotypes (from severe to mild) observed in FMF patients. Importantly, biparametric analyses discriminated also compound heterozygous FMF patients from HD with a sensitivity of $92.3 \%$ and a specificity of $100 \%$.

\section{DISCUSSION}

Here, we demonstrate that a functional assay, based on kinase inhibition and monitoring of pyroptosis and IL-1 $\beta$ release, accurately diagnoses FMF over a large number of $M E F V$ genotypes. This test discriminates FMF patients from both HD and patients with OID, including patients will well-defined monogenic diseases (supplementary figure 3). Besides the usual quantification of IL-1 family cytokine release, the test takes advantage of the real-time analysis of pyroptosis, a hallmark of inflammasome activation.

Cell death test brings first results within $3 \mathrm{~h}(2 \mathrm{~h}$ for sample preparation $+1 \mathrm{~h}$ for cell death analysis; $12 \mathrm{~h}$ for IL-1 $\beta$ ELISA) and costs less than $7.2 € /$ sample (supplementary table 2). Thus, it could be used in routine to support clinical findings. Due to its good positive predictive value, the test detects true positive FMF patients, for whom 
treatment could be initiated promptly, deep investigations re-evaluated, and genetic confirmation reached. On the other hand, its good negative predictive value indicates a robust way to identify true negative patients, who require further investigations, reasoned genetic testing, and postponed (or even no) colchicine initiation. Of note, biparametric analyses should be used whenever the test based on cell death leads to negative results in order to build confidence in the results.

Our test identifies FMF patients with both monoallelic and biallelic $M E F V$ mutations. These results are in line with clinical findings of patients with full-blown clinical FMF bearing monoallelic $M E F V$ mutations. Interestingly, and although the results need to be confirmed in larger cohorts, the in vitro monocyte responses to UCN-01 largely mimic clinical responses to colchicine $\mathrm{e}^{11,15}$ and the genotype-phenotype results. ${ }^{10,14,16,17}$ However, one limitation of our study stems from the fact that the results are mainly based on genetically-confirmed pathogenic mutations, thus requiring confirmatory analyses on larger cohorts of patients with rarer genotypes.

Our test uses isolated monocytes, preventing its transfer to routine labs in its current design. Nevertheless, we and others ${ }^{19}$ have preliminary data indicating that functional assays in whole blood are feasible and reliable, at least for cytokine release assessment (supplementary figure 4).

Altogether, functional assays are promising approaches for rapid detection of FMF patients with clearly-pathogenic mutations. ${ }^{19,20}$ Yet, the evaluation of pre-test probability (i.e. epidemiological and clinical data) remains the key in early decisions, thus positioning functional assays at the crossroads of clinics and genetics. 


\section{ACKNOWLEGMENTS}

The authors thank all the patients who took part in this study.

\section{FUNDING}

The study (Depist-FMF, registered at clinicaltrials.gov under the unique identifier NCT03747315) was founded by a grant from the Hospices Civils de Lyon (Jeune Chercheur HCL) and a grant from the Agence Nationale de la Recherche/Direction Générale de l'Offre de Soin (FMFgeneToDiag \#ANR-17-CE17-0021) and funding from the European Union's Horizon 2020 research and innovation program under grant agreement \#779295 (ImmunAID).

\section{CONFLICT OF INTEREST}

YJ, FM, AB, AM, and TH are co-inventors and owners of a patent "Methods and kits for diagnosis of familial Mediterranean fever" (WO/2019/048569).

\section{REFERENCES $(\max 20)$}

1. Özen S. Update on the epidemiology and disease outcome of Familial Mediterranean fever. Best Pract Res Clin Rheumatol 2018; 32: 254-260.

2. Ozen S, Karaaslan Y, Ozdemir O, et al. Prevalence of juvenile chronic arthritis and familial Mediterranean fever in Turkey: a field study. J Rheumatol 1998; 25: 2445-2449. 3. Zemer D, Pras M, Sohar E, et al. Colchicine in the prevention and treatment of the amyloidosis of familial Mediterranean fever. N Engl J Med 1986; 314: 1001-1005.

4. Gattorno M, Hofer M, Federici S, et al. Classification criteria for autoinflammatory recurrent fevers. Ann Rheum Dis 2019; 78: 1025-1032.

5. Ancient missense mutations in a new member of the RoRet gene family are likely to cause familial Mediterranean fever. The International FMF Consortium. Cell 1997; 90: 797-807. 
6. French FMF Consortium. A candidate gene for familial Mediterranean fever. Nat Genet 1997; 17: 25-31.

7. Jéru I, Hentgen V, Cochet E, et al. The Risk of Familial Mediterranean Fever in MEFV Heterozygotes: A Statistical Approach. PLoS ONE; 8. 2013. DOI: 10.1371/journal.pone.0068431.

8. Infevers - Tabular list, https://infevers.umai-montpellier.fr/web/search.php?n=1 (accessed 26 May 2020).

9. Magnotti F, Lefeuvre L, Benezech S, et al. Pyrin dephosphorylation is sufficient to trigger inflammasome activation in familial Mediterranean fever patients. EMBO Mol Med 2019; 11: e10547.

10. Touitou I, Magne X, Molinari N, et al. MEFV mutations in Behçet's disease. Hum Mutat 2000; 16: 271-272.

11. Wu Z, Zhang S, Li J, et al. Association between MEFV Mutations M694V and M680I and Behçet's Disease: A Meta-Analysis. PloS One 2015; 10: e0132704.

12. Federici S, Calcagno G, Finetti M, et al. Clinical impact of MEFV mutations in children with periodic fever in a prevalent western European Caucasian population. Ann Rheum Dis 2012; 71: 1961-1965.

13. Omenetti A, Carta S, Delfino L, et al. Increased NLRP3-dependent interleukin $1 \beta$ secretion in patients with familial Mediterranean fever: correlation with MEFV genotype. Ann Rheum Dis 2014; 73: 462-469.

14. Van Gijn ME, Ceccherini I, Shinar Y, et al. New workflow for classification of genetic variants' pathogenicity applied to hereditary recurrent fevers by the International Study Group for Systemic Autoinflammatory Diseases (INSAID). J Med Genet 2018; 55: 530-537.

15. Ryan JG, Masters SL, Booty MG, et al. Clinical features and functional significance of the P369S/R408Q variant in pyrin, the familial Mediterranean fever protein. Ann Rheum Dis 2010; 69: 1383-1388.

16. Cekin N, Akyurek ME, Pinarbasi E, et al. MEFV mutations and their relation to major clinical symptoms of Familial Mediterranean Fever. Gene 2017; 626: 9-13.

17. Gershoni-Baruch R, Brik R, Shinawi M, et al. The differential contribution of MEFV mutant alleles to the clinical profile of familial Mediterranean fever. Eur J Hum Genet EJHG 2002; 10: 145-149. 
18. Balta B, Erdogan M, Kiraz A, et al. A comprehensive molecular analysis and genotype-phenotype correlation in patients with familial mediterranean fever. Mol Biol Rep 2020; 47: 1835-1843.

19. Van Gorp H, Huang L, Saavedra P, et al. Blood-based test for diagnosis and functional subtyping of familial Mediterranean fever. Ann Rheum Dis. Epub ahead of print 20 April 2020. DOI: 10.1136/annrheumdis-2019-216701.

20. Shiba T, Tanaka T, Ida H, et al. Functional evaluation of the pathological significance of MEFV variants using induced pluripotent stem cell-derived macrophages. J Allergy Clin Immunol 2019; 144: 1438-1441.e12.

\section{FIGURES LEGENDS}

Figure 1. PKC inhibitors trigger fast cell death specifically in monocytes from FMF

patients. A-C Monocytes from HD (n=71), FMF patients bearing biallelic p.M694I/V variants $(n=38)$ or patients with OID $(n=40)$ were treated with $12.5 \mu$ M UCN-01. (A) Cell death was monitored in real time by measuring propidium iodide influx every 5 minutes. (B) The areas under the curve (AUC) were computed for each patient after 60 minutes of UCN-01 treatment. (C) The time required to reach $20 \%$ cell death was calculated for each HD, FMF and patients with OID. In the figure the values were normalized by subtracting the result obtained for the HD with the value obtained for the FMF/OID analyzed at the same time. (D-E) Receiver operating characteristic (ROC) curves were computed for the area under the cell death kinetics curve following UCN-01 treatment by comparing HD and FMF (D) and FMF and patients with OID (E). For each ROC curve, the AUC, specificity, sensitivity, as well as the positive (PPV) and the negative (NPV) predictive values are indicated.

Data information: (A) Each point of the curve corresponds to the average of the mean cell death values from three biological replicates of monocytes from the indicated patients. (B-C) Each dot represents the value from one patient. (A-B) The bar represents the $95 \%$ confidence interval. ${ }^{* * *} \mathrm{p}<0.001$ by Mann-Whitney rank-sum test.

Figure 2. Biparametric analysis discriminates FMF patients from HD or OID. (A) Monocytes from HD ( $n=71)$, FMF bearing biallelic p.M694I/V variants $(n=35)$, and patients with OID $(n=39)$ were primed with LPS during $3 \mathrm{~h}$ and treated with $12.5 \mu \mathrm{M}$ 
UCN-01 for $1.5 \mathrm{~h}$. IL-1 $\beta$ levels in monocyte supernatants were quantified by ELISA. (B-C) ROC curves were computed for the obtained IL-1 $\beta$ values by comparing FMF and HD (B) and FMF and patients with OID (C). (D) Cell death data and IL-1 $\beta$ data were combined by multiplying the mean area under the cell death kinetics curve by the concentrations of IL-1 $\beta$ for each patient. The log value is represented in the figure. (E-F) ROC curves were computed for the biparametric analysis data by comparing FMF and HD (E) and FMF and patients with OID (F). For each ROC curve, the AUC, specificity, sensitivity, as well as the positive (PPV) and the negative (NPV) predictive values are indicated.

Data information: (A, D) Each dot represents the mean value from three biological replicates for one patient (A) or the calculated value from one patient (D). (A, D) The bar represents the $95 \%$ confidence interval. *** $\mathrm{p}<0.001$ by Mann-Whitney rank-sum test.

Figure 3. UCN-01 treatment discriminates FMF heterozygous patients who present a gene dosage response. Monocytes from HD (n=71), FMF homozygous (p.M694IV/p.M694I-V) patients (Homo, n=38) and FMF heterozygous (p.M694I-V/0) patients (Het, $n=10$ ) were treated with $12.5 \mu \mathrm{M}$ UCN-01 either after LPS priming (C) or not (A). (A) Cell death was monitored in real time by measuring propidium iodide influx every 5 minutes. (B) The AUC were computed for each patient after 60 minutes of UCN-01 treatment. (C) IL-1 $\beta$ levels in monocyte supernatants were quantified by ELISA. (D) The two parameters were combined by multiplying the mean AUC by the concentration of IL-1 $\beta$ obtained for each patient.

Data information:

(A) Each point of the curve corresponds to the average of the mean cell death values from three biological replicates of monocytes from the indicated patients. (B-D) Each dot represents the value from one patient. (C) Each dot represents the mean value from three biological replicates for one patient. (A-D) The bar represents the 95\% confidence interval. ${ }^{*} \mathrm{p}<0.05,{ }^{* *} \mathrm{p}<0.01,{ }^{* * *}<0.001$ by Mann-Whitney rank-sum test. ns: not significant. 


\section{KEY MESSAGES:}

\section{What is already known about this subject?}

- Genetic analysis of the $M E F V$ gene is often inconclusive due to the large number of variants of uncertain significance.

\section{What does this study add?}

- Monitoring pyroptosis in real time allows a fast diagnosis of patients with familial Mediterranean fever.

- Monitoring both cell-death kinetics and IL-1 $\beta$ release accurately discriminates patients with familial Mediterranean fever from other patients.

- The nature and the number of the $M E F V$ variants influence the degree of in vitro activation of the pyrin inflammasome.

\section{How might this impact on clinical practice or future developments?}

- The test could guide early clinical decisions and management by identifying FMF patients who will require colchicine and genetic analyses while others (the negative ones) will need deeper investigations including the search for alternative diagnosis. 

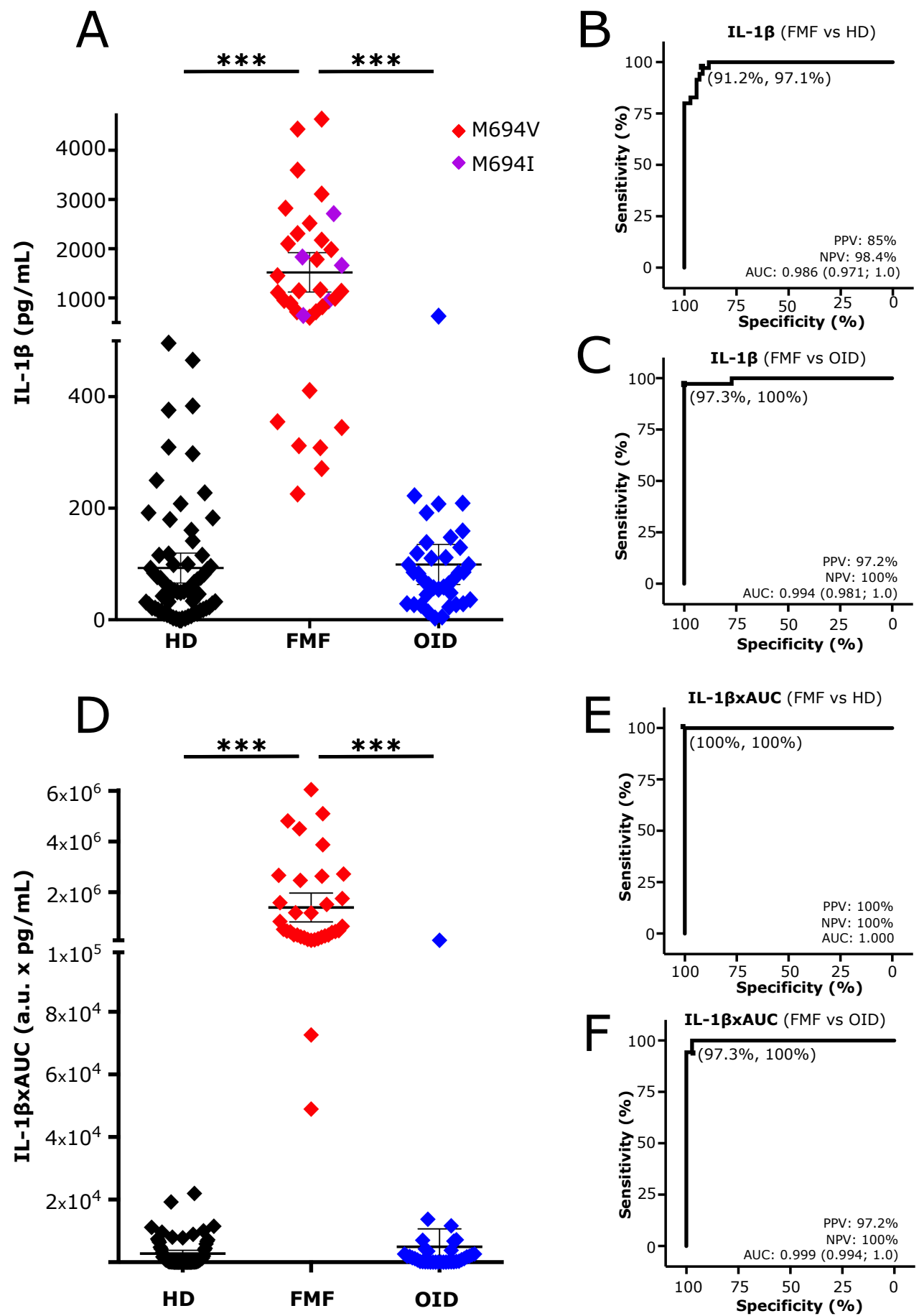

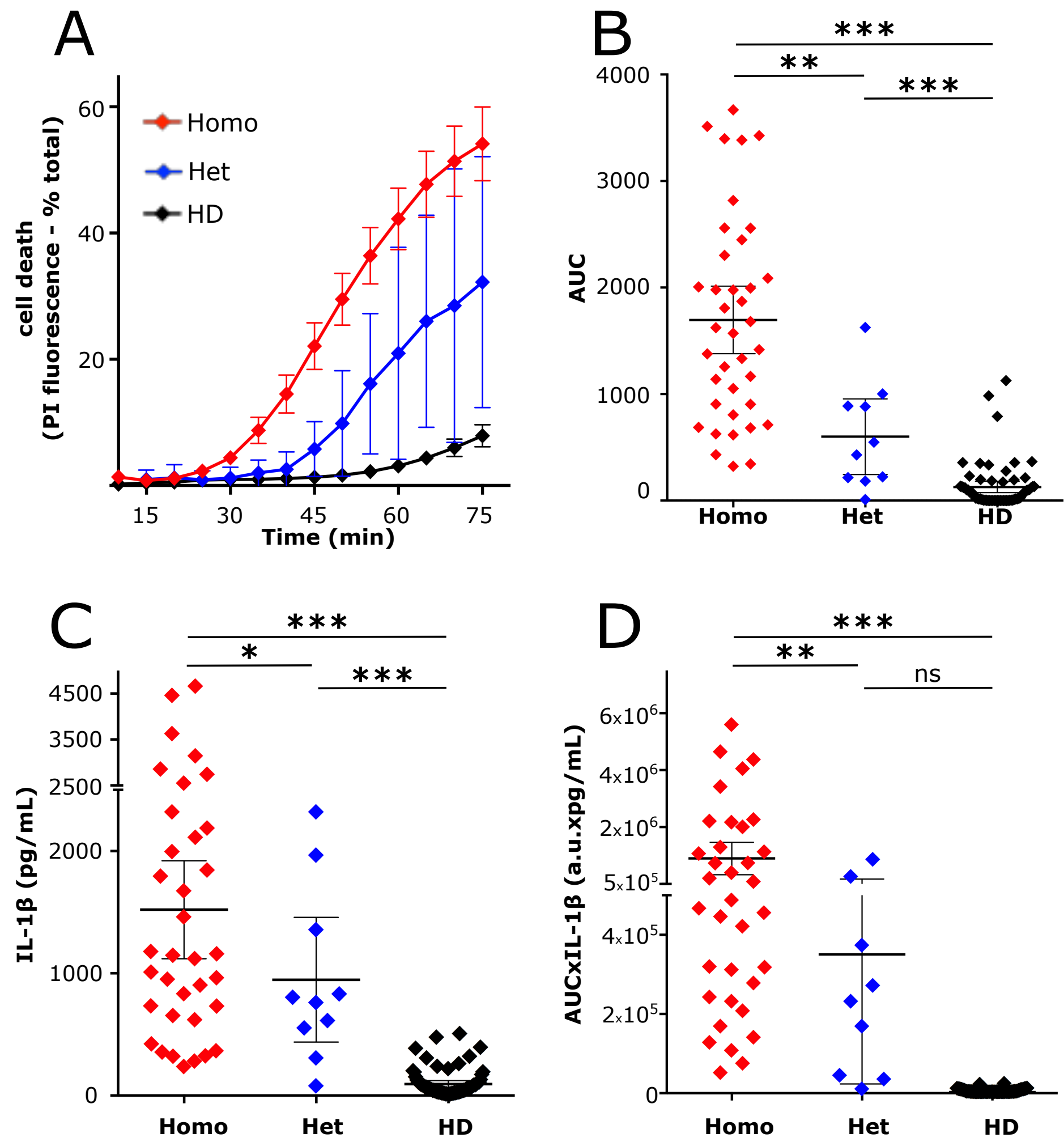
related to

by Flora Magnotti, Tiphaine Malsot, Sophie Georgin-Lavialle, Fatima Abbas, Amandine Martin, Alexandre Belot, Maxime Fauter, Muriel Rabilloud, Mathieu Gerfaud-Valentin, Pascal Sève, Agnès Duquesne, Arnaud Hot, Stéphane Durupt, Gilles Grateau, Thomas Henry, and Yvan Jamilloux

\section{MATERIALS AND METHODS}

\section{Patients and controls}

67 patients with symptomatic FMF and bearing at least one $M E F V$ mutation were prospectively included in the study (homozygous, $n=41$; compound heterozygous, $n=16$; heterozygous, $n=10$ ). The study was designed to include patients with symptomatic FMF (with past or current symptoms), which diagnosis was based on international criteria. Asymptomatic carriers of clearly pathogenic $M E F V$ mutations were no included. 71 healthy donors (HD) along with 40 patients suffering from other inflammatory disorders [i.e. adultonset Still's disease (AOSD, $n=6)$; systemic juvenile idiopathic arthritis (sJIA, $n=2)$; Behçet's disease $(n=4)$; Periodic Fever, Aphthous stomatitis, Pharyngitis, Adenitis (PFAPA, $n=2)$; inflammatory bowel disease (IBD, $n=4)$; sepsis $(n=3)$; Mevalonate Kinase Deficiency (MKD, $n=5)$; TNF-Receptor-Associated Periodic Syndrome (TRAPS, $n=2$ ); Cryopyrin-Associated Periodoc Syndrome $(n=2)$; systemic lupus erythematous (SLE, $n=2$ ); (non-systemic) polyarticular juvenile idiopathic arthritis (pJIA, $n=1$ ); H-syndrome 
$24(n=1)$; haploinsufficiency of A20 (HA20, $n=1$ ); fever of unknown origin (FUO, $n=4)$; sarcoidosis $(\mathrm{n}=1)]$; were used as controls (see patient description in supplementary table 1). Except those with a genetically-determined disease (i.e. MKD, TRAPS, CAPS, HA20, Hsyndrome), patients with OID did not undergo systematic genetic investigation.

\section{Monocyte isolation}

Blood was drawn in heparin-coated tubes and kept at room temperature overnight. Blood samples from HD were drawn on the same day as patients. Peripheral blood mononuclear cells (PBMCs) were isolated by density-gradient centrifugation using Lymphocyte Separation Medium (Eurobio). Monocytes were isolated from PBMCs by magnetic selection using CD14 MicroBeads (Miltenyi Biotec) and the AutoMACS Pro Separator (Miltenyi Biotec) following the manufacturer's instructions. Monocytes were enumerated in the presence of a viability marker (propidium iodide (PI), $10 \mu \mathrm{g} / \mathrm{ml}$, Sigma) by flow cytometry (BD Accuri C6 Flow Cytometer).

\section{Pyrin inflammasome activation and cell death / IL-1ß secretion analyses}

Monocytes were seeded in Roswell Park Memorial Institute (RPMI) 1640, GlutaMAX medium (Thermo Fisher Scientific) supplemented with 10\% fetal calf serum (Lonza). Cell death was monitored by incubating $2 \times 10^{4}$ monocytes per well in black 96 -well plates (Costar, Corning) in the presence of PI at $5 \mu \mathrm{g} / \mathrm{ml}$. Three technical replicates per condition were done. UCN-01 was added at $12.5 \mu \mathrm{M}$ in the absence of any priming signal. After UCN01 addition, real-time PI incorporation was measured every 5 min from 15 to 75 min postUCN intoxication, using a fluorimeter (Tecan) at the following wavelengths: excitation 535 
$47 \mathrm{~nm}$ (bandwidth $15 \mathrm{~nm}$ ) and emission $635 \mathrm{~nm}$ (bandwidth $15 \mathrm{~nm}$ ). Cell death was normalized using PI incorporation in monocytes treated with Triton X-100 for 15 min

49 (corresponding to the $100 \%$ cell death).

For the IL-1ß secretion analysis, monocytes were seeded onto 96 -well plates at $5 \times 10^{3}$

51 cells/well, incubated for $3 \mathrm{~h}$ in the presence of lipopolysaccharide (LPS, $10 \mathrm{ng} / \mathrm{ml}$,

52 InvivoGen) and then treated with UCN-01 (12.5 $\mu \mathrm{M}$, Sigma) during $1.5 \mathrm{~h}$. Following the

53 incubation, plates were centrifuged and supernatants were collected. IL-1ß levels were

54 quantified by ELISA (R\&D Systems) following the manufacturer's instructions.

55

56 Whole blood test analysis

$571 \mathrm{~mL}$ of blood from heparin-coated tubes was diluted with $1 \mathrm{~mL}$ Phosphate Buffered Saline 58 (PBS, ThermoFisher Scientific). The diluted blood was transferred into heparin-coated 59 tubes with plasma-separating gel (BD368497) and incubated for $1 \mathrm{~h}$ at $37^{\circ} \mathrm{C}$ in the 60 presence of LPS (10 ng/mL). UCN-01 was then added at $12.5 \mu \mathrm{M}$ final. Following a $1.5 \mathrm{~h}$ 61 incubation, the tube was centrifuged (10 $\mathrm{min}-1500 \mathrm{~g})$ and the plasma was collected for IL$621 \beta$ dosage by ELISA (R\&D Systems) following the manufacturer's instructions.

63

64 Ethical approval information

65 The study was approved by the French Comité de Protection des Personnes (CPP,\#L16-189) 66 and by the French Comité Consultatif sur le Traitement de l'Information en matière de 67 Recherche dans le domaine de la Santé (CCTIRS, \#16.864). The experiments conformed to 68 the principles set out in the WMA Declaration of Helsinki and the Department of Health and 69 Human Services' Belmont Report. The Etablissement Français du Sang provided HD blood 
70 in the framework of the convention \#14-1820. Informed consent was received from each

71 participant prior to inclusion in the study.

72 All data are available within the manuscript, supplementary material, or on request to the

73 authors. DEPIST-FMF study is registered on www.clinicaltrials.gov under the unique id $74 \quad$ \#NCT03747315.

76 Patient and Public Involvement Statement

77 Patients were not involved in the design, recruitment or in the conduct of the study. Results 78 will be disseminated through communications to the French FMF patient association 79 (AFFMF) and through the FMF and AID Global Association website.

\section{Statistical analyses}

82 Patients and HD data normal distribution was verified using D'Agostino-Pearson omnibus 83 normality test. Quantitative data are presented as means $( \pm$ standard deviation in the text, 84 or with the $95 \%$ confidence interval in the figures, as indicated). Triplicate reliability was 85 verified by calculating intraclass correlation coefficients.

86 The ability of the test to discriminate patients with FMF from HD or subjects with OID was 87 quantified by the construction of ROC curves and the estimation of areas under the curve 88 (AUC) with their associated 95\%-confidence intervals (95\%-CI). Differences in mean values 89 between groups were also tested using an analysis of variance (ANOVA and repeated 90 ANOVA). Differences with p-values $<0.05$ were considered statistically significant. p-value $91<0.05,<0.01$ and, $<0.001$ are marked by ${ }^{*},{ }^{* *}$ and, ${ }^{* * *}$, respectively. 
92 ROC curves and AUC estimates were made using the non-parametric method of DeLong.

93 The threshold was estimated by maximizing the Youden index (Youden Index: Se + Sp-1).

94 Sensitivity, specificity, positive and negative predictive values were calculated using this 95 threshold.

96 For cell death kinetic analysis, the best time point for discriminating FMF patients from HD 97 was searched by modelling ROC curve according to time (Parametric Distribution Free 98 [PDF] approach). Using the estimated best time point, the optimal threshold (Youden 99 Index) and the associated sensitivity, specificity, positive and negative predictive values 100 were assessed.

101 To calculate the time leading to $20 \%$ cell death, a non-linear regression analysis was used 102 to fit a sixth-order polynomial curve to the normalized cell death kinetics using the least 103 squares fit as the fitting method. The obtained curve was used to interpolate the time 104 corresponding to $20 \%$ cell death. The subtractions of (FMF or OID) patients - HD were then 105 plotted on violin-plots using R software. The biparametric test is based on multiplication of 106 the AUC at 60 minutes (AUC60) value obtained from the cell death kinetic modelisation by 107 the concentration of IL-1 $\beta$. The result is expressed as arbitrary unit x picogram / millilitre 108 (a.u. x pg / mL).

109 Statistical analyses were carried out with Prism (GraphPad, v6) and R software (R 110 Foundation for Statistical Computing, v4.0, http://www.R-project.org).

\section{References for statistical analyses}

113 DeLong ER. Comparing the areas under two or more correlated receiver operating characteristic 114 curves: a a nonparametric approach. Biometrics. 1988; 44(3):837-45

115 Mc Clish DK. Analyzing a portion of the ROC curve. Med Decis Making. 1989; 9(3):190-5 
116 Youden WJ. Index for rating diagnostic tests'. Cancer. 1950; 3:32-35

117 Perkins NJ et al. The inconsistency of optimal cutpoints obtained using two criteria based on the 118 Receiver Operating Characteristic curve. Am J Epidemiol. 2006;163(7):670-675

119 Todd AA. Distribution-free ROC analysis using binary regression techniques. Biostatistics. 120 2002;3(3): 421-432

121 Pepe MS. A regression modelling framework for Receiver Operating Characteristic curves in 122 medical diagnostic testing. Biometrika. 1997;84:595-608 


\section{SUPPLEMENTARY FIGURES}

125

126

supplementary figure 1

127
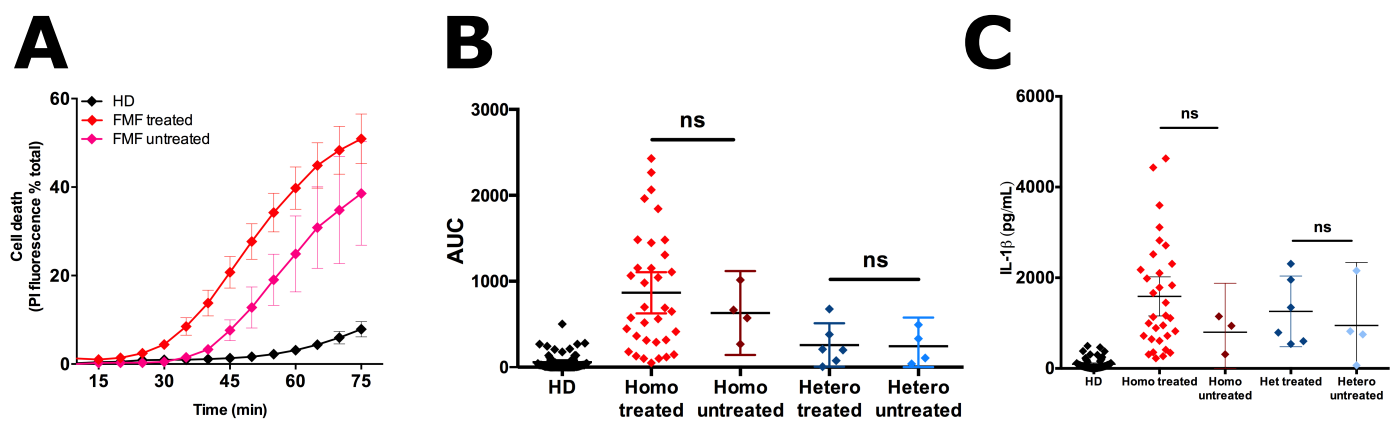

128

129 Supplementary figure 1: impact of colchicine treatment on UCN-based test. 

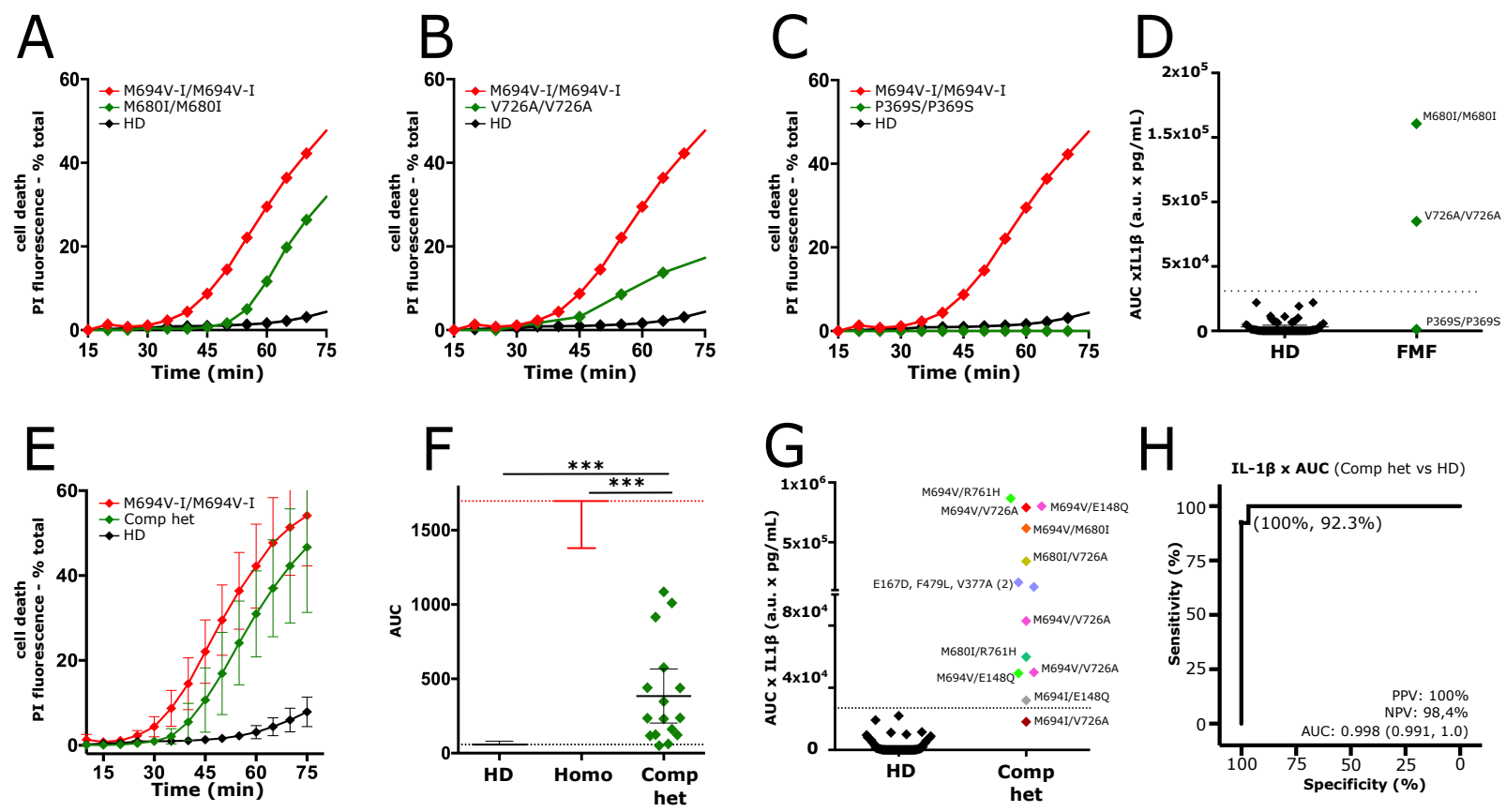

Supplementary figure 2: MEFV genotype influences monocyte responses to UCN-01. (A-C) Cell death kinetics was obtained by monitoring the propidium iodide influx every 5 minutes in monocytes from HD ( $\mathrm{n}=71$ ), FMF homozygous patients (carrying the p.M694I-V/p.M694I-V, $n=38$ ), one patient with the p.M680I/p.M680I mutation (A), one patient with the p.V726A/ p.V726A mutation (B) and one patient with the p.P369S/ p.P369S mutation (C). (D) The mean AUC value for each experiment was combined with the corresponding concentration of IL- $1 \beta$ for each patient to perform the biparametric analysis. (E) Cell death kinetics curves were obtained from monocytes from HD (n=71), FMF homozygous patients (p. M694I-V, $\mathrm{n}=38$ ) and compound heterozygous FMF patients (Comp het, $\mathrm{n}=16$ ). (F) AUC values for each patient were computed after 60 minutes of UCN-01 treatment. (G) Mean AUC and IL-1 $1 \beta$ release of 13 patients were combined for the biparametric analysis. $(\mathrm{H})$ ROC curves were calculated for the biparametric analysis data by comparing Comp het FMF patients and HD.

143 Data information: (A-C, E) Each point of the curve corresponds to the average of the mean cell death values 144 from three biological replicates of monocytes from the indicated patients. (D, F-G) Each dot represents the value from one patient. The bar represents the $95 \%$ confidence interval. ${ }^{* * *} \mathrm{p}<0.001$ by Mann-Whitney ranksum test. 
supplementary figure 3

A

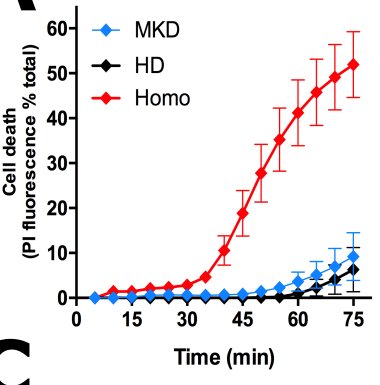

60- $\rightarrow$ TRAPS

歌 $50-\rightarrow \mathrm{HD}$

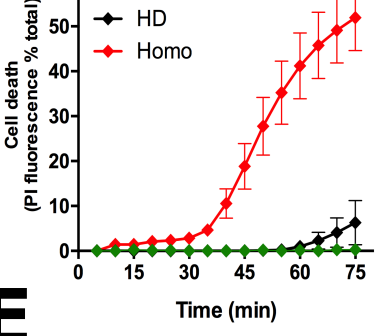

$\mathbf{E}$

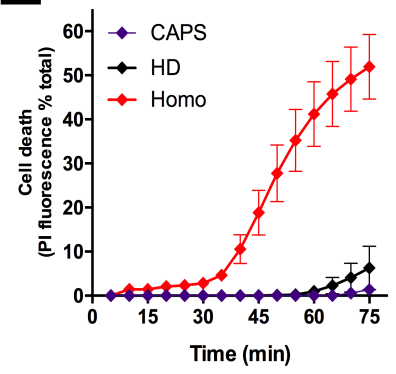

B

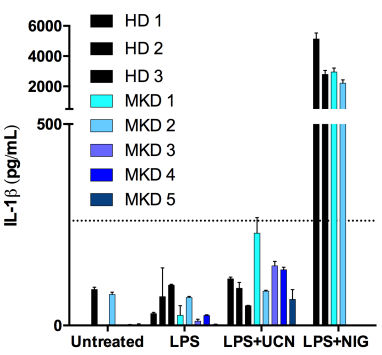

D

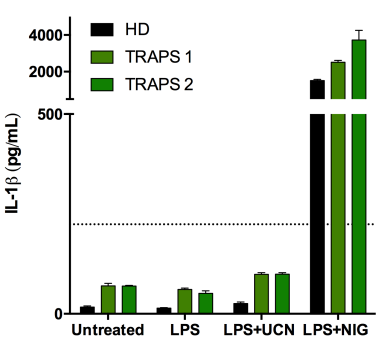

E

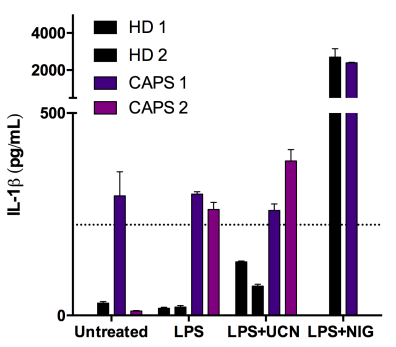

Supplementary figure 3: UCN-based test combined with ELISA discriminates FMF patients from patients with monogenic diseases. Cell death kinetics was obtained by monitoring the propidium iodide influx every 5 minutes in monocytes from HD (n=8), patients with MKD (n=5) (A), TRAPS (n=2) (C) or CAPS $(n=2)(E) .(B, D, F)$ IL-1 $\beta$ concentrations were analyzed in patients monocytes treated or not with LPS alone (3h), LPS+UCN-01 (UCN, 1.5h) or LPS+nigericin (NIG, 1.5h).

Data information: $(\mathrm{A}, \mathrm{C}, \mathrm{E})$ Each point of the curve corresponds to the average of the mean cell death values from three biological replicates of monocytes from the indicated patients. (B,D,F) Each histogram represents the mean value of a triplicate from one patient. The bar represents the SEM; the dotted line is set at the threshold determined in figure 1, discriminating homozygous (M694V/I) FMF patients and HD. that does not significantly increase in the presence of UCN-01. Importantly, in the absence of LPS, neither constitutive pyroptosis nor UCN-01-induced pyroptosis is detected in CAPS patients monocytes (E). Patients 
162 with very rare monogenic diseases linked to pyrin inflammasome or cytoskeleton disorders (e.g. PAPA, PFIT 163 and PAAND patients) could not be included in this study.

164

165

166

167

168 


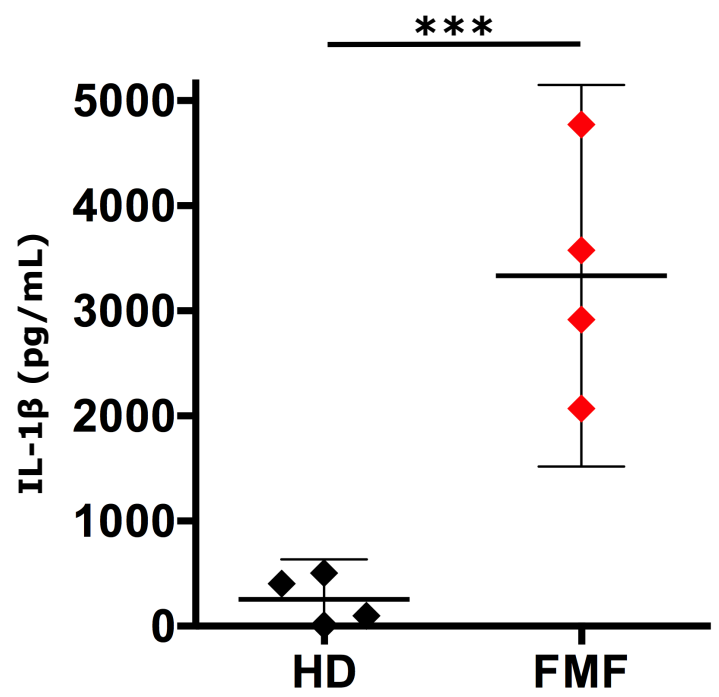

171

172

173 Supplementary figure 4: UCN-01-based inflammasome test in whole blood. Whole blood samples from

174 HD $(n=4)$ and homozygous FMF patients (p.M694I-V, n=4) were primed with LPS and treated with UCN-01 in 175 plasma-separating gel tubes. After centrifugation, plasma IL-1 $\beta$ concentrations were determined by ELISA.

176 Each dot represents the mean value from three biological replicates for one patient. The bar represents the $17795 \%$ confidence interval. ${ }^{* * *} \mathrm{p}<0.001$ by Mann-Whitney rank-sum test.

178 


\section{Supplementary table 1: Description of patients and controls included in the study}

\begin{tabular}{|c|c|c|c|c|c|c|}
\hline \# & $\begin{array}{c}\text { Sex } \\
\text { (Male/Female) }\end{array}$ & $\begin{array}{c}\text { Age } \\
\text { (years) }\end{array}$ & Disease & MEFV genotype & $\begin{array}{l}\text { Colchicine } \\
\text { at time of } \\
\text { sampling }\end{array}$ & $\begin{array}{c}\text { Symptoms at time of } \\
\text { sampling }\end{array}$ \\
\hline 1 & $\mathrm{~F}$ & 43 & FMF & M694V/M694V & yes & no \\
\hline 2 & M & 34 & FMF & M694V/M694V & yes & no \\
\hline 3 & M & 5 & FMF & M694V/M694V & yes & no \\
\hline 4 & $\mathrm{~F}$ & 11 & FMF & M694V/M694V & yes & no \\
\hline 5 & $\mathrm{~F}$ & 13 & FMF & M694V/M694V & yes & no \\
\hline 6 & $\mathrm{~F}$ & 9 & FMF & M694V/M694V & yes & no \\
\hline 7 & $\mathrm{~F}$ & 5 & FMF & M694V/M694V & yes & no \\
\hline 8 & $\mathrm{~F}$ & 8 & FMF & M694V/M694V & no & no \\
\hline 9 & $\mathrm{~F}$ & 7 & FMF & M694V/M694V & yes & no \\
\hline 10 & $\mathrm{~F}$ & 15 & FMF & M694V/M694V & yes & no \\
\hline 11 & M & 4 & FMF & M694V/M694V & yes & no \\
\hline 12 & $\mathrm{~F}$ & 17 & FMF & M694V/M694V & yes & no \\
\hline 13 & M & 63 & FMF & M694V/M694V & yes & no \\
\hline 14 & M & 9 & FMF & M694V/M694V & yes & no \\
\hline 15 & M & 4 & FMF & M694V/M694V & yes & no \\
\hline 16 & M & 21 & FMF & M694V/M694V & no & no \\
\hline 17 & M & 17 & FMF & M694V/M694V & yes & no \\
\hline 18 & $\mathrm{~F}$ & 44 & FMF & M694V/M694V & yes & no \\
\hline 19 & $\mathrm{~F}$ & 72 & FMF & M694V/M694V & yes & no \\
\hline 20 & M & 44 & FMF & M694V/M694V & yes & no \\
\hline 21 & M & 63 & FMF & M694V/M694V & yes & no \\
\hline 22 & M & 35 & FMF & M694V/M694V & yes & no \\
\hline 23 & $\mathrm{~F}$ & 74 & FMF & M694V/M694V & yes & no \\
\hline 24 & M & 71 & FMF & M694V/M694V & yes & no \\
\hline 25 & M & 33 & FMF & M694V/M694V & yes & no \\
\hline 26 & $\mathrm{~F}$ & 43 & FMF & M694V/M694V & yes & no \\
\hline 27 & $\mathrm{~F}$ & 40 & FMF & M694V/M694V & yes & no \\
\hline 28 & M & 73 & FMF & M694V/M694V & yes & no \\
\hline 29 & M & 19 & FMF & M694V/M694V & yes & no \\
\hline 30 & M & 32 & FMF & M694V/M694V & yes & no \\
\hline 31 & $\mathrm{~F}$ & 10 & FMF & M694V/M694V & yes & no \\
\hline 32 & M & 22 & FMF & M694V/M694V & yes & yes \\
\hline 33 & M & 30 & FMF & M694I/M694I & yes & no \\
\hline 34 & M & 16 & FMF & M694I/M694I & yes & no \\
\hline 35 & $\mathrm{~F}$ & 37 & FMF & M694I/M694I & yes & no \\
\hline 36 & M & 40 & FMF & M694I/M694I & no & no \\
\hline 37 & $\mathrm{~F}$ & 54 & FMF & M694I/M694I & yes & yes \\
\hline 38 & M & 11 & FMF & M694I/M694I & no & no \\
\hline
\end{tabular}




\begin{tabular}{|c|c|c|c|c|c|c|}
\hline 39 & $\mathrm{~F}$ & 32 & FMF & M680I/M680I & yes & no \\
\hline 40 & $\mathrm{~F}$ & 35 & FMF & V726A/V726A & yes & no \\
\hline 41 & $\mathrm{~F}$ & 13 & FMF & P369S/P369S & yes & no \\
\hline 42 & M & 15 & FMF & M694V/0 & yes & no \\
\hline 43 & M & 5 & FMF & M694V/0 & no & no \\
\hline 44 & M & 43 & FMF & M694V/0 & no & no \\
\hline 45 & $\mathrm{~F}$ & 44 & FMF & M694V/0 & no & no \\
\hline 46 & $\mathrm{~F}$ & 25 & FMF & M694V/0 & no & no \\
\hline 47 & M & 5 & FMF & $\mathrm{M} 694 \mathrm{~V} / 0$ & yes & no \\
\hline 48 & M & 40 & FMF & M694V/0 & yes & no \\
\hline 49 & M & 41 & FMF & M694I/0 & yes & no \\
\hline 50 & $\mathrm{~F}$ & 7 & FMF & M694I/0 & yes & no \\
\hline 51 & M & 56 & FMF & M694I/0 & yes & no \\
\hline 52 & $\mathrm{~F}$ & 10 & FMF & M694V/M680I & yes & no \\
\hline 53 & $\mathrm{~F}$ & 8 & FMF & M680I/V726A & yes & no \\
\hline 54 & $\mathrm{~F}$ & 36 & FMF & M680I/R761H & yes & no \\
\hline 55 & $\mathrm{~F}$ & 8 & FMF & M694V/R761H & yes & no \\
\hline 56 & M & 5 & FMF & $\begin{array}{c}\text { E167D,F479L;V37 } \\
\text { 7A }\end{array}$ & yes & no \\
\hline $\mathbf{5 7}$ & M & 13 & FMF & $\begin{array}{c}\text { E167D,F479L;V37 } \\
\text { 7A }\end{array}$ & yes & no \\
\hline 58 & M & 8 & FMF & E148Q/M694V & yes & no \\
\hline 59 & $\mathrm{~F}$ & 30 & FMF & M694I/V726A & yes & yes \\
\hline 60 & M & 12 & FMF & M694I/E148Q & yes & no \\
\hline 61 & $\mathrm{~F}$ & 22 & FMF & M694V/M680I & yes & no \\
\hline 62 & M & 51 & FMF & M694V/V726A & yes & no \\
\hline 63 & M & 55 & FMF & M694V/V726A & yes & no \\
\hline 64 & $\mathrm{~F}$ & 41 & FMF & M694V/R761H & yes & no \\
\hline 65 & $\mathrm{~F}$ & 30 & FMF & M694V/R761H & yes & no \\
\hline 66 & $\mathrm{H}$ & 38 & FMF & M694V/E148Q & yes & no \\
\hline 67 & M & 40 & FMF & M694V/V726A & yes & no \\
\hline 1 & M & 17 & AOSD & NA & no & no \\
\hline 2 & M & 53 & AOSD & NA & yes & no \\
\hline 3 & $\mathrm{~F}$ & 53 & AOSD & NA & no & no \\
\hline 4 & $\mathrm{~F}$ & 19 & AOSD & NA & no & no \\
\hline 5 & $\mathrm{~F}$ & 19 & AOSD & NA & no & no \\
\hline 6 & $\mathrm{~F}$ & 19 & AOSD & NA & no & yes \\
\hline 7 & $\mathrm{~F}$ & 32 & Behçet & NA & yes & no \\
\hline 8 & $\mathrm{M}$ & 34 & Behçet & NA & yes & no \\
\hline 9 & $\mathrm{~F}$ & 28 & Behçet & NA & yes & no \\
\hline 10 & $\mathrm{~F}$ & 32 & Behçet & NA & no & no \\
\hline 11 & $\mathrm{~F}$ & 17 & IBD & NA & no & no \\
\hline 12 & $\mathrm{~F}$ & 17 & IBD & NA & no & no \\
\hline
\end{tabular}




\begin{tabular}{|c|c|c|c|c|c|c|}
\hline 13 & $\mathrm{M}$ & 17 & IBD & $\mathrm{NA}$ & no & no \\
\hline 14 & M & 13 & IBD & NA & no & no \\
\hline 15 & M & 43 & SLE & NA & no & no \\
\hline 16 & M & 47 & SLE & NA & no & no \\
\hline 17 & $\mathrm{~F}$ & 6 & pJIA & NA & no & no \\
\hline 18 & $\mathrm{~F}$ & 28 & Sepsis & NA & no & no \\
\hline 19 & $\mathrm{~F}$ & 27 & Sepsis & NA & no & no \\
\hline 20 & M & 89 & Sepsis & NA & no & no \\
\hline 21 & $\mathrm{~F}$ & 13 & SJIA & NA & no & no \\
\hline 22 & $\mathrm{~F}$ & 16 & sJIA & NA & no & no \\
\hline 23 & M & 3 & MKD & NA & no & no \\
\hline 24 & $\mathrm{~F}$ & 11 & MKD & NA & no & no \\
\hline 25 & M & 5 & MKD & NA & no & no \\
\hline 26 & M & 4 & MKD & NA & no & $\begin{array}{l}\text { yes (fever, abdominal } \\
\text { pain, elevated CRP) }\end{array}$ \\
\hline 27 & M & 20 & MKD & NA & no & $\begin{array}{l}\text { yes (stomatitis, } \\
\text { elevated CRP) }\end{array}$ \\
\hline 28 & M & 26 & HA20 & NA & no & no \\
\hline 29 & $\mathrm{~F}$ & 4 & H-syndrome & NA & no & no \\
\hline 30 & $\mathrm{~F}$ & 36 & TRAPS & NA & no & no \\
\hline 31 & $\mathrm{~F}$ & 38 & TRAPS & NA & no & no \\
\hline 32 & $\mathrm{~F}$ & 19 & CAPS & NA & no & no \\
\hline 33 & M & 49 & CAPS & NA & no & no \\
\hline 34 & $\mathrm{~F}$ & 17 & FUO & NA & no & yes \\
\hline 35 & $\mathrm{~F}$ & 22 & PFAPA & NA & yes & no \\
\hline 36 & M & 41 & PFAPA & NA & yes & no \\
\hline 37 & M & 44 & FUO & NA & no & no \\
\hline 38 & M & 37 & FUO & NA & no & no \\
\hline 39 & M & 48 & FUO & NA & no & no \\
\hline 40 & M & 42 & Sarcoidosis & NA & no & no \\
\hline 1 & M & 26 & HD & NA & no & no \\
\hline 2 & $\mathrm{M}$ & 58 & HD & NA & no & no \\
\hline 3 & $\mathrm{~F}$ & 30 & HD & NA & no & no \\
\hline 4 & $\mathrm{M}$ & 22 & HD & NA & no & no \\
\hline 5 & M & 25 & HD & NA & no & no \\
\hline 6 & $\mathrm{M}$ & 28 & HD & NA & no & no \\
\hline 7 & $\mathrm{~F}$ & 51 & HD & NA & no & no \\
\hline 8 & $\mathrm{M}$ & 53 & HD & NA & no & no \\
\hline 9 & M & 55 & HD & NA & no & no \\
\hline 10 & $\mathrm{M}$ & 32 & HD & NA & no & no \\
\hline 11 & M & 51 & HD & NA & no & no \\
\hline 12 & $\mathrm{~F}$ & 32 & HD & NA & no & no \\
\hline 13 & M & 61 & HD & NA & no & no \\
\hline
\end{tabular}




\begin{tabular}{|c|c|c|c|c|c|c|}
\hline 14 & $\mathrm{M}$ & 63 & HD & NA & no & no \\
\hline 15 & $\mathrm{M}$ & 18 & HD & NA & no & no \\
\hline 16 & $\mathrm{~F}$ & 30 & HD & NA & no & no \\
\hline 17 & $\mathrm{~F}$ & 21 & HD & NA & no & no \\
\hline 18 & $\mathrm{~F}$ & 31 & HD & NA & no & no \\
\hline 19 & $\mathrm{M}$ & 38 & HD & NA & no & no \\
\hline 20 & $\mathrm{M}$ & 34 & HD & NA & no & no \\
\hline 21 & $\mathrm{M}$ & 31 & HD & NA & no & no \\
\hline 22 & $\mathrm{M}$ & 34 & HD & NA & no & no \\
\hline 23 & $\mathrm{M}$ & 41 & HD & NA & no & no \\
\hline 24 & $\mathrm{~F}$ & 53 & HD & NA & no & no \\
\hline 25 & M & 40 & HD & NA & no & no \\
\hline 26 & $\mathrm{M}$ & 33 & HD & NA & no & no \\
\hline 27 & $\mathrm{~F}$ & 25 & HD & NA & no & no \\
\hline 28 & $\mathrm{~F}$ & 41 & HD & NA & no & no \\
\hline 29 & $\mathrm{M}$ & 38 & HD & NA & no & no \\
\hline 30 & $\mathrm{M}$ & 40 & HD & NA & no & no \\
\hline 31 & M & 47 & HD & NA & no & no \\
\hline 32 & $\mathrm{M}$ & 54 & HD & NA & no & no \\
\hline 33 & $\mathrm{M}$ & 48 & HD & NA & no & no \\
\hline 34 & $\mathrm{M}$ & 45 & HD & NA & no & no \\
\hline 35 & $\mathrm{M}$ & 51 & HD & NA & no & no \\
\hline 36 & $\mathrm{~F}$ & 63 & HD & NA & no & no \\
\hline 37 & $\mathrm{M}$ & 58 & HD & NA & no & no \\
\hline 38 & $\mathrm{M}$ & 60 & HD & NA & no & no \\
\hline 39 & $\mathrm{M}$ & 40 & HD & NA & no & no \\
\hline 40 & $\mathrm{M}$ & 62 & HD & NA & no & no \\
\hline 41 & $\mathrm{M}$ & 58 & HD & NA & no & no \\
\hline 42 & $\mathrm{~F}$ & 51 & HD & NA & no & no \\
\hline 43 & $\mathrm{M}$ & 45 & HD & NA & no & no \\
\hline 44 & $\mathrm{M}$ & 18 & HD & NA & no & no \\
\hline 45 & $\mathrm{M}$ & 41 & HD & NA & no & no \\
\hline 46 & $\mathrm{M}$ & 53 & HD & NA & no & no \\
\hline 47 & $\mathrm{M}$ & 43 & HD & NA & no & no \\
\hline 48 & $\mathrm{M}$ & 26 & HD & NA & no & no \\
\hline 49 & $\mathrm{~F}$ & 64 & HD & NA & no & no \\
\hline 50 & $\mathrm{~F}$ & 29 & HD & NA & no & no \\
\hline 51 & $\mathrm{M}$ & 61 & HD & NA & no & no \\
\hline 52 & M & 24 & HD & NA & no & no \\
\hline 53 & $\mathrm{~F}$ & 43 & HD & NA & no & no \\
\hline 54 & $\mathrm{M}$ & 31 & HD & NA & no & no \\
\hline 55 & $\mathrm{~F}$ & 21 & HD & NA & no & no \\
\hline
\end{tabular}




\begin{tabular}{lllllll}
\hline $\mathbf{5 6}$ & M & 63 & HD & NA & no & no \\
$\mathbf{5 7}$ & M & 31 & HD & NA & no & no \\
$\mathbf{5 8}$ & M & 59 & HD & NA & no & no \\
$\mathbf{5 9}$ & M & 64 & HD & NA & no & no \\
$\mathbf{6 0}$ & M & 62 & HD & NA & no & no \\
$\mathbf{6 1}$ & F & 43 & HD & NA & no & no \\
$\mathbf{6 2}$ & M & 60 & HD & NA & no & no \\
$\mathbf{6 3}$ & F & 40 & HD & NA & no & no \\
$\mathbf{6 4}$ & M & 37 & HD & NA & no & no \\
$\mathbf{6 5}$ & M & 62 & HD & N/A & no & no \\
$\mathbf{6 6}$ & M & 55 & HD & N/A & no & no \\
$\mathbf{6 7}$ & F & 27 & HD & N/A & no & no \\
$\mathbf{6 8}$ & M & 40 & HD & N/A & no & no \\
$\mathbf{6 9}$ & M & 53 & HD & N/A & no & no \\
$\mathbf{7 0}$ & F & 42 & HD & N/A & n/A & no \\
$\mathbf{7 1}$ & M & 38 & HD & & &
\end{tabular}


187 Supplementary Table 2: Estimate of the costs of the functional assays

188

\begin{tabular}{|c|c|c|c|c|}
\hline \multirow{2}{*}{ PRODUCT } & \multicolumn{2}{|c|}{ STOCK } & \multicolumn{2}{|l|}{ PER PATIENT } \\
\hline & Quantity & Price & Quantity & Price \\
\hline Ficoll & $500 \mathrm{ml}$ & $47 €$ & $10 \mathrm{ml}$ & $0.94 €$ \\
\hline CD14 beads & $2 \mathrm{ml}$ & $560 €$ & $20 \mu \mathrm{l}$ & $5.60 €$ \\
\hline AutoMACS columns & 10 & $499 €$ & - & $0.49 €$ \\
\hline LPS & $5 \mathrm{mg}$ & $146 €$ & $10 \mathrm{ng} / \mathrm{ml}(0.5 \mu \mathrm{l} /$ experiment $)$ & $0.07 €$ \\
\hline UCN-01 & $5 \mathrm{mg}$ & $724 €$ & $12.5 \mu \mathrm{M}(1 \mu \mathrm{l} /$ experiment $)$ & $0.07 €$ \\
\hline Propidium Iodide & $100 \mathrm{mg}$ & $129 €$ & $5 \mu \mathrm{g} / \mathrm{ml}(7.5 \mu \mathrm{l} /$ experiment $)$ & $0,09 €$ \\
\hline IL-1 $\beta$ ELISA KIT & 25 plates & $521 €$ & 12 wells & $2.17 €$ \\
\hline & & & Total for cell death assay & $7.19 €$ \\
\hline & & & Total with ELISA & $9.43 €$ \\
\hline
\end{tabular}

189

190 